\title{
EchoGéo
}

$46 \mid 2018$

Varia

\section{Paris, Berlin, Shanghai}

Jean-Louis Chaléard

\section{OpenEdition}

Journals

\section{Édition électronique}

URL : https://journals.openedition.org/echogeo/16298

DOI : 10.4000/echogeo.16298

ISSN : 1963-1197

\section{Éditeur}

Pôle de recherche pour l'organisation et la diffusion de l'information géographique (CNRS UMR 8586)

Référence électronique

Jean-Louis Chaléard, «Paris, Berlin, Shanghai », EchoGéo [En ligne], 46 | 2018, mis en ligne le 31 décembre 2018, consulté le 31 juillet 2021. URL : http://journals.openedition.org/echogeo/16298 ; DOI : https://doi.org/10.4000/echogeo.16298

Ce document a été généré automatiquement le 31 juillet 2021.

EchoGéo est mis à disposition selon les termes de la licence Creative Commons Attribution - Pas d'Utilisation Commerciale - Pas de Modification 4.0 International (CC BY-NC-ND) 


\title{
Paris, Berlin, Shanghai
}

\author{
Jean-Louis Chaléard
}

1 Les varia ont leur diversité interne propre. En même temps, chaque année, la livraison proposée est différente de la précédente par les thèmes abordés comme par l'importance relative des rubriques. Un point commun fréquent pourtant: la prépondérance des textes sur la ville ou des questions urbaines; cette fois-ci, les grandes agglomérations l'emportent, de la banlieue parisienne à Berlin, en passant par Shanghai, confirmant l'importance des études urbaines en géographie, particulièrement des métropoles. Autre sujet souvent abordé: les questions environnementales, ici à travers leur dimension sociale que ce soit dans la production des savoirs, la gestion des crises ou leurs conséquences sur la santé.

Cinq articles alimentent la rubrique Sur le champ. Deux portent sur la région parisienne, un sur Shanghai, c'est-à-dire sur des questions urbaines ; leurs entrées sont cependant très différentes et à des degrés divers originales : la santé, les populations reléguées, le chant... A. Croisé, A. Sierra et B. Lysianuk s'interrogent sur les risques sanitaires liés aux héritages industriels, fort nombreux, de Seine-Saint Denis. Ils analysent la visibilité, immédiate ou tardive, d'une dégradation de la santé des travailleurs et des riverains d'aménagements industriels dans le département. Ils soulignent que les transformations urbaines actuelles tendent à valoriser les sites, dans le cadre de ce qu'il a appellent le « recyclage urbain ", contribuant à rendre invisibles les conséquences sanitaires des activités productives.

M. Flégeau étudie un projet d'aménagement de la plaine de Pierrelaye, en périphérie parisienne, cherchant à transformer cette plaine agricole polluée, délaissée et occupée par des Gens du voyage et des Roms, en une forêt aménagée. Elle relève la difficile prise en compte des populations marginalisées et met en évidence la part d'instrumentalisation dont fait l'objet le projet pour se défaire des occupations illégales, appuyé par un argumentaire paysager et social.

4 Dans un tout autre contexte, S. Defoin-Merlin propose une analyse de Shanghai par ses lieux de chant. En Chine, chanter est une activité courante et appréciée. Elle est aussi structurante. Ainsi, la répartition et l'architecture des lieux de chant illustrent-elles les politiques culturelles et l'aménagement urbain. Leur temporalité, leur fréquentation et 
leur fonctionnement sont des indices de la structuration sociale de la métropole. Les lieux de chant révèlent à la fois la polycentralité urbaine et de nouvelles dynamiques sociales à l'œuvre dans la ville.

5 Les deux autres textes proposent des sujets portant sur des questions à la fois environnementales, sociales et politiques. A. Sérandour s'intéresse à la construction et la diffusion des savoirs autour de l'exploitation du lithium, dans les trois principaux producteurs d'Amérique latine, Argentine, Bolivie et Chili. Elle montre que la multiplication des projets d'exploitation s'accompagne d'une production croissante de savoirs par les universitaires nationaux sur le lithium, sans toutefois que ces nouveaux acquis soient mis à profit par les multinationales minières. Celles-ci développent leurs connaissances et leurs innovations techniques à l'étranger dans le cadre de réseaux transnationaux tandis que les savoirs produits localement, loin d'être sans effet, jouent un rôle social et politique participant à l'intégration du gisement à d'autres échelles : régionale, nationale, locale.

6 T. Nicolas, F. Pagey Bénito-Espinal, É. Lagahé et M.-L. Gobinddass revisitent la question des catastrophes cycloniques à partir de l'impact des ouragans majeurs de septembre 2017 dans la Caraïbe insulaire. Ils montrent que le facteur principal de vulnérabilité des sociétés caribéennes face à ces catastrophes n'est pas systématiquement lié à la puissance des ouragans ou au niveau de pauvreté, mais beaucoup plus aux structures fondamentales des sociétés insulaires. La politique menée depuis plus d'un demi-siècle par Cuba témoigne de la nécessité de délier la vulnérabilité de la pauvreté. De même, les dysfonctionnements enregistrés à Saint-Martin (partie française de l'île) ou à Porto Rico, invitent à repenser le lien entre l'appartenance à une grande nation et le niveau de vulnérabilité, et à s'intéresser aux maux sociétaux qui conduisent à la catastrophe.

7 La rubrique Sur le vif est particulièrement fournie ce trimestre puisqu'elle s'est enrichie de trois articles, qui portent sur des sujets différents, tant par leur domaine que dans leur rapport à l'actualité immédiate. En mai 2018, l'INSEE a décidé d'intégrer l'économie de la drogue dans le calcul du PIB. C'est l'occasion pour A.Slim de s'interroger sur l'intérêt du PIB, sa signification, les questions de comparaison, en plus des redoutables problèmes méthodologiques posés par son calcul.

8 En cette année de coupe du monde de football remportée par l'équipe de France, A. Langar, M. Baron et C Grasland s'interrogent sur la situation du football tunisien à partir d'un article paru dans le journal Le Monde. Le football tunisien est intimement lié à l'histoire et la géographie du pays. Il s'enracine dans les bouleversements qu'a connus le pays depuis le Protectorat et a joué un grand rôle dans les luttes politiques. Il s'adosse mais aussi contribue en partie à entretenir les profondes inégalités territoriales, notamment entre le littoral et l'intérieur, le Nord et le Sud.

$9 \quad$ L'article le plus récent porte sur les élections législatives italiennes du 4 mars 2018 dont les conséquences sont importantes, non seulement à l'échelle italienne, mais aussi au niveau international, par les changements qu'elles provoquent dans les rapports entre l'Italie et l'extérieur, notamment l'Union européenne. M. Cavallaro, D. Policastro, G. Salza et M. A. Zanetti proposent une analyse géographique du scrutin. Ils mettent en évidence les évolutions territoriales et sociales de l'Italie, à travers la répartition des votes. Apparaissent ainsi trois Italie, avec trois électorats différents : un centre-gauche qui résiste dans les grands centres urbains, une Ligue forte dans les villes de petite 
taille, particulièrement du Nord, et le M5S (Mouvement 5 étoiles), parti transversal, qui progresse fortement dans le Sud.

Dans la rubrique Sur le métier, É. Bonnet-Pineau interviewe T. Wieder, correspondant du Monde à Berlin. C'est l'occasion pour le journaliste de présenter ce que lui a apporté et lui apporte encore la géographie dans son métier et d'analyser l'évolution de la ville sur le plan spatial et social, avec la chute du mur, les projets d'aménagement ambitieux, l'actualité migratoire... On revient au cœur des questions urbaines, voire plus spécifiquement métropolitaines, dans une capitale qui fut longtemps divisée et qui est réputée tant pour sa scène artistique que pour ses réalisations monumentales et urbanistiques.

\section{AUTEUR}

\section{JEAN-LOUIS CHALÉARD}

Jean-Louis Chaléard est Professeur des Universités, Université Paris I Panthéon Sorbonne et directeur de publication d'EchoGéo. 